\title{
A PROPERTY OF ORDERED RINGS
}

\section{A. A. ALBERT ${ }^{1}$}

In this note we shall provide the last essential element in a simple proof of the theorem of Wagner [1] which states that every ordered ring satisfying a nontrivial polynomial identity is commutative.

It is clear that an ordered ring $A$ has no divisors of zero. Moreover, the existence of a nontrivial identity is easily seen to imply that if $a \neq 0$ and $b \neq 0$ are in $A$ there exist elements $c \neq 0$ and $d \neq 0$ in $A$ such that

$$
a c=b d .
$$

Hence $A$ is what O. Ore [2] has called a regular ring. Then $A$ can be imbedded in a unique quotient ring $B$. Every element of $B$ can be expressed as a product

$$
\alpha=b^{-1} a,
$$

for $b \neq 0$ and $a$ in $A$. By (1) we can always write

$$
\alpha=b^{-1} a=d c^{-1} \text {. }
$$

Since $\alpha=(-b)^{-1}(-a)=(-d)(-c)^{-1}$ we can assume that, in the case where $A$ is ordered, the denominators $b$ and $c$ are always positive. We now derive the following sequence of simple lemmas.

Lemma 1. Let $\alpha=b^{-1} a=d c^{-1}$ where $b>0, c>0$. Then $a$ and $d$ have the same sign.

For $b^{-1} a=d c^{-1}$ if and only if $b d=a c$. Since $b>0$ and $c>0$ the elements $b d$ and $a c$ of $A$ can be equal only if $a$ and $d$ have the same sign.

Lemma 2. Let $\alpha=b^{-1} a=c^{-1} f$ where $b>0, c>0$. Then $a$ and $f$ have the same sign.

For we use (3) to write $\alpha=d c^{-1}$ where $c>0$, and $d$ has the same sign as $a$. By Lemma 1 we know that $f$ has the same sign as $d$ and hence the same sign as $a$.

Since the sign of $a$ is unique we may say that $\alpha=b^{-1} a>0$ if $a>0$, $\alpha<0$ if $a<0, \alpha=0$ if $a=0$. We may then prove the following result.

Received by the editors March 1, 1956.

1 This paper was sponsored in part by the Office of Ordnance Research, United States Army, under Contract No. DA-11-022-ORD-1571. 
LeMmA 3. Let $\alpha \neq 0$ be in $B$ and let there exist positive elements $a$ and $b$ of $A$ such that $c=a \alpha b$ is in $A$. Then $c$ and $\alpha$ have the same sign.

For $a \alpha=c b^{-1}$ has the same sign as $c$ by our definition of sign. Also $c b^{-1}=e^{-1} f$ where $e>0$ and $f$ has the same sign as $c$. Then $\alpha=(e a)^{-1} f$, $e a>0, \alpha$ has the same sign as $f$ and hence the same sign as $c$.

Lemma 4. Let $\alpha$ and $\beta$ be in $B$ and $\alpha>0, \beta>0$. Then $\alpha+\beta$ and $\alpha \beta$ are positive.

For we may write $\alpha=a^{-1} b, \beta=d c^{-1}$ with $a, b, c, d$ all positive. Then $a(\alpha+\beta) c=a\left(a^{-1} b+d c^{-1}\right)=b c+a d>0$. By Lemma 3 we have $\alpha+\beta>0$. Also $a(\alpha \beta) c=(a \alpha)(\beta c)=b d>0$ and so $\alpha \beta>0$.

If $\alpha<0$ and $\beta<0$ then $-\alpha>0,-\beta>0,(-\alpha)(-\beta)=\alpha \beta>0$. Similarly if $\alpha<0$ and $\beta>0$ we have $-(\alpha \beta)=(-\alpha) \beta>0$ and $\alpha \beta<0$. We have completed a proof of the following result.

THEOREM. The quotient ring of an ordered regular ring is ordered.

As a consequence of results of Amitsur [3] and Kaplansky [4] we have the property which states that if an ordered ring $A$ satisfies a nontrivial polynomial identity the quotient ring $B$ also satisfies the identity and is finite-dimensional over its center $F$. By our theorem $B$ is ordered and this order clearly implies that $F$ is ordered. But then it is known [5] that $B$ is commutative and so we have Wagner's result that $A$ is commutative.

\section{REFERENCES}

1. Über die Grundlagen der projectiven Geometrie und allgemeine Zahlensysteme, Math. Ann. vol. 113 (1937) pp. 528-567.

2. Linear equations in non-commutative fields, Ann. of Math. vol. 32 (1931) pp. 463-471.

3. On rings with identities, J. London Math. Soc. vol. 30 (1955) pp. 464-470. 580 .

4. Rings with a polynomial identity, Bull. Amer. Math. Soc. vol. 54 (1948) pp. 575-

5. A. A. Albert, On ordered algebras, Bull. Amer. Math. Soc. vol. 46 (1940) pp. 521-522.

The University of Chicago 